\title{
Erratum to: Candidate Genes Associated with Susceptibility for SARS-Coronavirus
}

Ying-Hen Hsieh ${ }^{\mathrm{a}, *}$, Cathy W.S. Chen ${ }^{\mathrm{b}}$, Shu-Fang Hsu Schmitz ${ }^{\mathrm{c}}$, Chwan-Chuan King ${ }^{\mathrm{d}}$, Wei-Ju Chen ${ }^{\mathrm{e}}$, Yi-Chun $\mathrm{Wu}^{\mathrm{f}}$, Mei-Shang $\mathrm{Ho}^{\mathrm{e}}$

${ }^{a}$ Department of Public Health and Biostatistics Center, China Medical University, Taichung, Taiwan 402

${ }^{b}$ Department of Statistics, Feng Cha University, Taichung, Taiwan

${ }^{c}$ Institute of Mathematical Statistics, University of Bern, Bern, Switzerland

${ }^{d}$ Institute of Epidemiology, College of Public Health, National Taiwan University, Taipei, Taiwan

${ }^{e}$ Institute of Biomedical Sciences, Academia Sinica, Taipei, Taiwan

${ }^{f}$ Department of Health, Center for Disease Control, Taipei, Taiwan

Published online: 5 February 2010

(C) Society for Mathematical Biology 2010

\section{Erratum to: Bulletin of Mathematical Biology (2010) 72:122-132 DOI 10.1007/s11538-009-9440-8}

In the published article the affiliations of the second author and the third author were erroneously reversed. The authors' affiliations should have read as seen above.

\footnotetext{
*Corresponding author.

E-mail address: hsieh@mail.cmu.edu.tw (Ying-Hen Hsieh).

The online version of the original article can be found under doi:10.1007/s11538-009-9440-8.
} 